\title{
Pressure-induced Polymerization and Electrical Conductivity of a Polyiodide
}

\author{
Tomasz Poręba, ${ }^{[a, b]}$ Michelle Ernst, ${ }^{[b]}$ Dominik Zimmer, ${ }^{[c]}$ Piero Macchi* ${ }^{* b, d]}$ and Nicola Casati ${ }^{*[a]}$
}

\begin{abstract}
We report the high-pressure structural characterization of an organic polyiodide salt in which a progressive addition of iodine to triiodide groups occurs. Compression leads to the initial formation of discrete heptaiodide units, followed by polymerization to a 3D anionic network. Although the structural changes appear to be continuous, the insulating salt becomes a semiconducting polymer above $10 \mathrm{GPa}$. The features of the pre-reactive state and the polymerized state are revealed by analysis of the computed electron and energy densities. The unusually high electrical conductivity can be explained with the formation of new bonds.
\end{abstract}

Polyiodides (PI) in crystal form were discovered over 190 years ago. ${ }^{[1]}$ Their structural diversity, due to the bonding flexibility of iodine, remains the subject of constant interest. ${ }^{[2-4]}$ Nowadays, various $\mathrm{PI}$ are known, ranging from $\mathrm{I}_{3}{ }^{-}$to $\mathrm{I}_{29}{ }^{3-}$, with the general formula: $I^{n-}{ }_{2 m+n}$, up to $n=4$. Their building blocks consist of $I_{3}{ }^{-}$as a donor and $\mathrm{I}_{2}$ as an acceptor forming a charge transfer (CT) complex. CT complexes can interact further to form extended structures with topologies up to cubic. Because the aggregation of iodine units is somewhat unpredictable, the design of Pls is not easy. Moreover, the classification of higher units is, so far, pragmatically based on the distance between iodine atoms, but universal criteria are still lacking.

Different theoretical approaches to study bonds in PI exist, e.g. calculations of the potential energy surface and bond order ${ }^{[5]}$, the energy decomposition analysis (EDA) ${ }^{[6]}$, the analysis of the electron density and its Laplacian at bond critical points (BCP $)^{[7]}$, the electron localization function, and the one-electron potential ${ }^{[8]}$. Furthermore, the energy density at $\mathrm{BCP}^{[9]}$ and the interacting quantum atom (IQA) method ${ }^{[10]}$ were used to analyze halogen bonds ${ }^{[11]}$.

Depending on the forces exerted by the organic counter ions (chemical pressure), PI exhibit useful electrochemical properties, like charge-carrier transportation, high electrolyte energy density,

[a] T, Poreba; Dr. N. Casati

Laboratory for Synchrotron Radiation - Condensed Matter

Paul Scherrer Institute

5232 Villigen - PSI

E-mail: nicola.casati@psi.ch

[b] T. Poreba; M. Ernst; Prof. P. Macchi

Department of Chemistry and Biochemistry

University of Bern

Freiestrasse 3, 3012 Bern

[c] Dr. D. Zimmer

Institute of Geosciences

Goethe-University Frankfurt

Crystallography/Mineralogy

Altenhoeferallee 1

60438 Frankfurt am Main

[d] Prof. P. Macchi

Department of Chemistry, Materials and Chemical Engineering

Polytechnic of Milan

Via Mancinelli 7, 20131 Milano

e-mail: piero.macchi@polimi.it high redox reaction reversibility ${ }^{[12,13]}$, and a wide range of electrical conductivity, from insulator to metallic behavior ${ }^{[14,15]}$. Hence, PI found technical applications in electronic and electrochemical devices such as flow batteries, fuel cells, dyesensitized solar cells and optical devices. ${ }^{[12,16,17]}$

Compared with the chemical pressure, an external mechanical pressure more substantially affects the crystal interand intramolecular landscape. In fact, a huge lattice strain may induce phase transformations and even chemical reactions. The achievable static high pressure (HP) in standard diamond anvil cells (DACs) is tens of gigapascals. Such a pressure changes significantly the Gibbs energy, increasing the internal energy and making the $P V$ term dominant. Similarly large energy changes are, instead, not accessible in solids by temperature alteration which induces instead sublimation or melting due to the dominant entropic term. The volume contraction is the main driving force for the addition reaction to atoms linked by unsaturated bonds and formation of extended assemblies, otherwise unstable at ambient pressure. One can apply this concept also to polyvalent iodine, typically able to expand its coordination in heptaiodides or organic iodanes ${ }^{[18]}$. Although compression may produce exotic chemical bonding and properties, only few spectroscopic studies focused on PI at HP. Raman spectroscopy demonstrated that solid organic $\mathrm{PI}$ under $\mathrm{HP}$ can catenate. Pentaiodides form $\mathrm{I}_{3}{ }^{-}$chains in iodinedoped poly(vinyl alcohol) films at as low as $0.2 \mathrm{GPa}{ }^{[19]}$, while a pressure between 0 and $3.4 \mathrm{GPa}$ is needed for bond formation in PI confined into carbon nanotubes ${ }^{[20]}$. Such a catenation does not occur in aqueous solution, advocating solid phase as a preferred matrix for a reaction between iodine units.

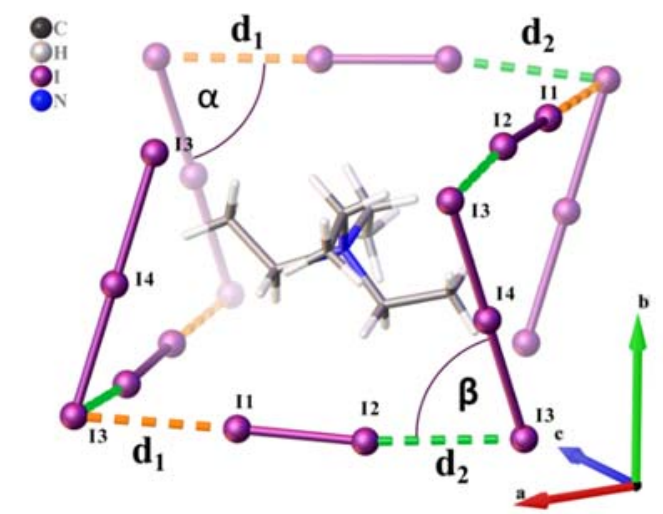

Figure 1: The iodine units around the tetraethylammonium cation in TEAl at ambient pressure. The shortest $\left(d_{1}=3.479(4) \AA\right)$ iodine-iodine intermolecular distances are marked in orange and the second shortest $\left(d_{2}=3.494(4) \AA\right)$ in green.

This document is the accepted manuscript version of the following article:

Por ba, T., Ernst, M., Zimmer, D., Macchi, P., \& Casati, N. (2019). Pressure-induced polymerization and electrical conductivity of a polyiodide. Angewandte Chemie International Edition, 58(20), 6625-6629. https://doi.org/10.1002/anie.201901178 

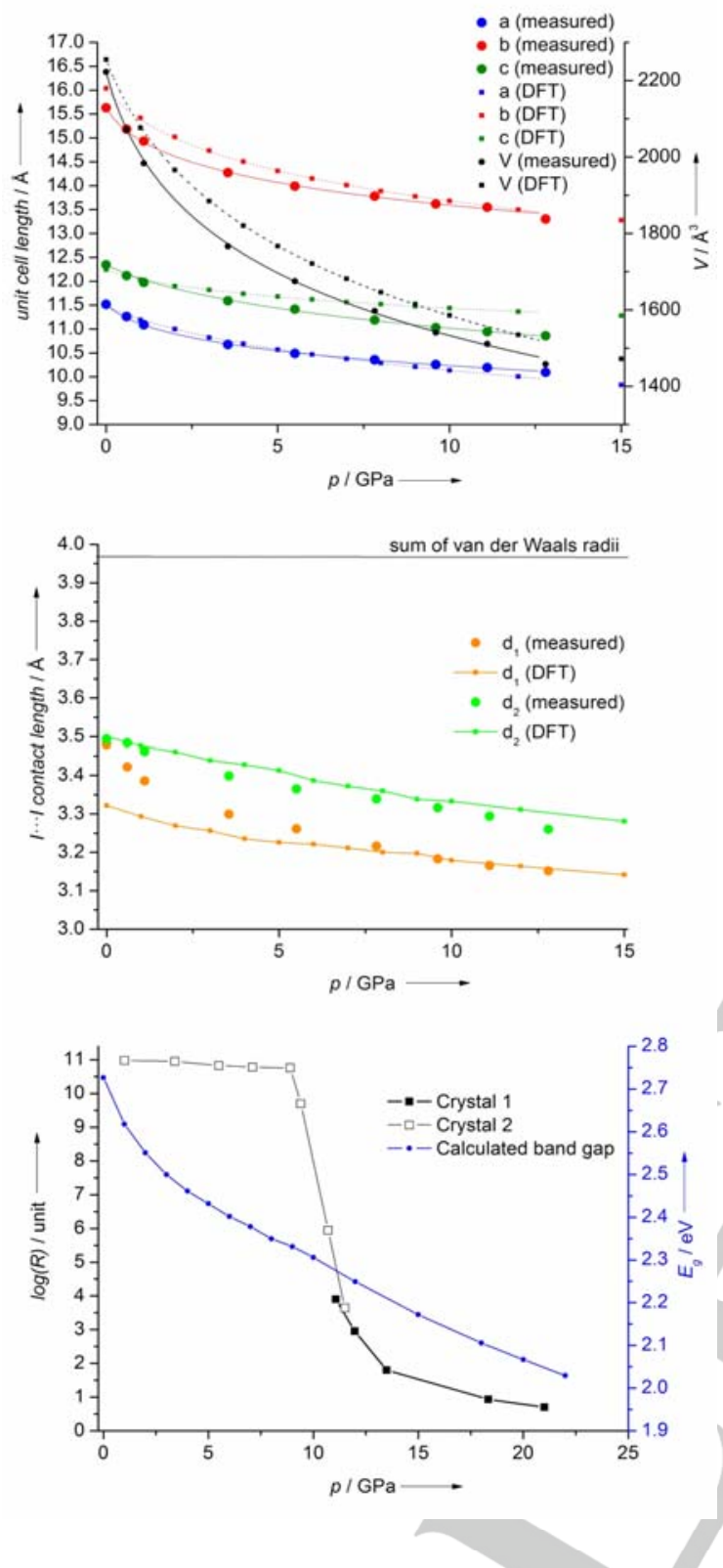

Figure 2: a) Unit cell axes and volume of TEAl as a function of pressure. Lines represent $3^{\text {rd }}$ order Vinet equation of state ${ }^{[21]}$ (fitted using EoSfit7 $\mathrm{c}^{[22]}$ ). The calculated Bulk Modulus is 7.7(5) GPa, which coincides with that of pure $\mathrm{I}_{2}{ }^{[23]}$. b) Evolution of $d_{1}$ and $d_{2}$ (see Fig. 1) with pressure. c) Electric resistance (measured on two different samples) and calculated band gap.

In this study, we investigated the response of tetraethylammonium di-iodine triiodide (TEAI) to compression, using powder and single-crystal X-ray diffraction, electrical conductivity, and first principle calculations. TEAI was chosen after extensive screening of the Cambridge Structural Database ${ }^{[24]}$ (entries HALTAP ${ }^{[25]}$ and HALTAP01 ${ }^{[26]}$ ) in order to find a moiety with appropriate packing, ready for catenation between donor $\left(\mathrm{I}_{3}{ }^{-}\right)$and acceptor $\left(\mathrm{I}_{2}\right)$ upon compression. Quaternary ammonium salts are often used as model cations for
PI studies ${ }^{[27-30]}$. At ambient conditions, TEAI crystallizes in the orthorhombic Aba2 space-group. $\mathrm{I}_{2}$ and apical iodine atoms of the $\mathrm{I}_{3}{ }^{-}$anion form, through contacts $\mathrm{d}_{1}$ and $\mathrm{d}_{2}$ (Fig. 1), zig-zag chains extending along the crystallographic $c$ axis, while oscillating in a direction. The same contacts form another zig-zag motif propagating along $a$ and oscillating mainly along the $b$ axis. These supramolecular chains encapsulate the isolated cations. Parallel chains form bilayers normal to the $b$ axis due to the linkage with $\mathrm{I}_{3}{ }^{-}$spacers (Fig. S2). The distances between one $I_{2}$ and its neighboring two $\mathrm{I}_{3}^{-}$units are unequal ( $d_{1}$ and $d_{2}$, Fig. 1 and $2 b$ ). They approach the acceptor at angles ( $\alpha$ and $\beta$, respectively) favorable for formation of higher units. This happens when the $I_{3}$ and $I_{2}$ are close enough for their frontier molecular orbitals to overlap. In extended systems, this interplay gives rise to valence and conducting bands ${ }^{[31]}$. When the interaction is stronger, the bands broaden and the band gap decreases, leading to a semiconducting behavior, observed in many species ${ }^{[32-34]}$.

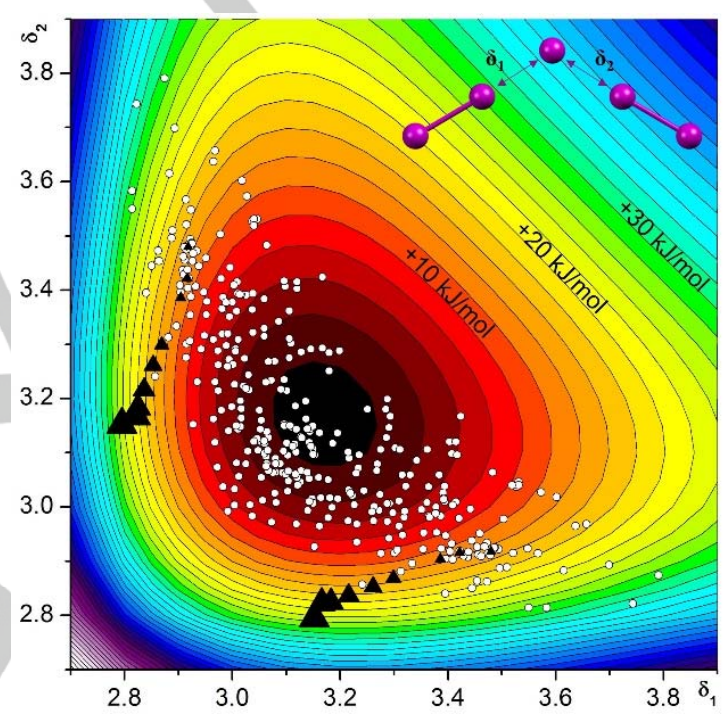

Figure 3: The electronic energy of $\mathrm{I}_{5}{ }^{-}$calculated in vacuo, as a function of $\delta_{1}$ and $\delta_{2}$. The colour indicates the destabilization from the symmetric $1_{5}$. The white circles represent entries in the Cambridge Structural Database containing $\mathrm{I}_{5}$. The black triangles represent TEAl at different pressures (the triangle size is proportional to the pressure).

We performed X-ray diffraction experiments on TEAI in various pressure transmitting media (PTM). Herein, we report data obtained using nitrogen as PTM, known to be hydrostatic up to ca. $10 \mathrm{GPa}{ }^{[35]}$ and chemically inert. The effects of other, less hydrostatic PTM, will be discussed in a forthcoming full paper. Upon pressurization up to $12.8 \mathrm{GPa}$, the crystal volume of TEAI contracts by $35 \%$. The $b$ axis, normal to the corrugated iodine bilayers, shows the highest linear compressibility, due to a lack of strong intermolecular interactions in this direction. Molecular volume compression trends (Fig. 2a) do not show any discontinuity ruling out the occurrence of phase transitions. The length of the unit cell axes $a$ and $b$ are well predicted by periodic density functional theory (DFT) calculations over the entire pressure regime whereas $c$ is slightly overestimated. The length of contact $d_{2}$ is well simulated whereas the underestimation of $d_{1}$ at low pressure is due to neglecting temperature effects in the 
simulations, whereas all high-pressure experiments have been carried out at ambient temperature. The temperature effects on the lattice parameters were estimated by an additional diffraction experiment at $110 \mathrm{~K}$ and ambient $\mathrm{P}$ (Table S4). Moreover, in the vicinity of the equilibrium geometry, the calculated energy surface is very flat (see Fig. 3 for $I_{5}^{-}$), which is the reason why some DFT functionals or basis sets may fail in predicting the geometry of the adducts, especially when perturbed by the electric field inside a crystal.

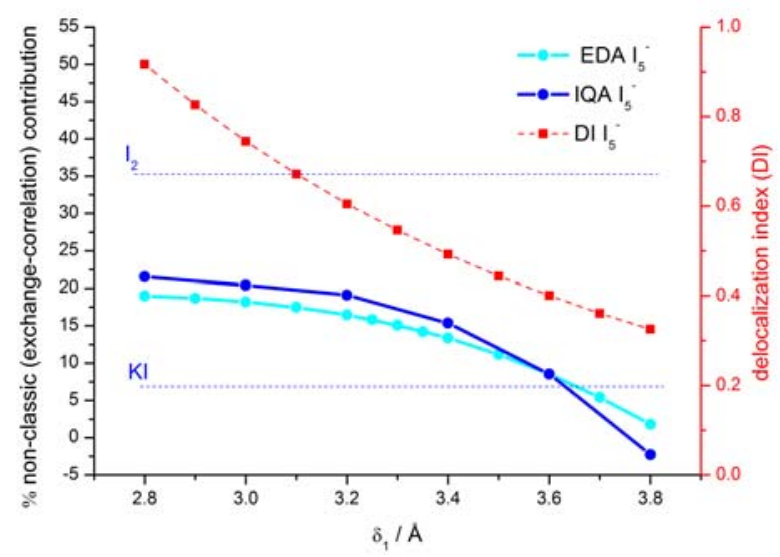

Figure 4: Bonding contribution in $I_{5}{ }^{-}$as a function of $\delta_{1}$ : the delocalization index is in red; the non-classic contributions to the binding energy (IQA or EDA) are in blue. The ionic $\mathrm{KI}$ and the covalent $\mathrm{I}_{2}$ are shown as benchmarks. The reference states for the decomposition calculations are: $\mathrm{I}^{-}+\mathrm{K}^{+} \rightarrow \mathrm{KI}, 1 \cdot+1 \cdot \rightarrow$ $\mathrm{I}_{2}, \mathrm{I}_{2}+\mathrm{I}_{3^{-}}, \rightarrow \mathrm{I}_{5^{-}}$. IQA and EDA decomposition for $\mathrm{I}_{3}{ }^{-}$is shown in Fig $\mathrm{S} 6$ of the SI.

Perpendicular to the iodine bilayers, the compression induces a continuous decrease of $\alpha$ and $\beta$ (Fig. 1 and Fig. S3). The contact $d_{2}$, subtending the $\beta$ angle, is less compressible than $d_{1}$, subtending $\alpha$ (Fig. S3). As the pressure increases, differences between the respective distances $d_{1}$ and $d_{2}$ as well as $\alpha$ and $\beta$ broaden. This forces the iodine zig-zag motif to bend out of its initial shape. It is illustrated by a gradual increase of torsion between triiodides (Fig. S3). Compression of the intermolecular iodine bonds does not cause any notable distortion of the intramolecular geometry of the $\mathrm{I}_{2}$ and $\mathrm{I}_{3}{ }^{-}$units.

In order to gain more insight into the nature of the bond in PI, we used theoretical tools for bonding analysis. In TEAI, various interactions of PI units occur among themselves and with the cation, which complicates the analysis. For simplification and abstraction, we take $\mathrm{I}_{5}{ }^{-}$calculated in vacuo as benchmark. The stable structure of $\mathrm{I}_{5}^{-}$has $\mathrm{C}_{2 \mathrm{v}}$ symmetry, which can be seen as two $\mathrm{I}_{2}$ molecules symmetrically interacting with a central $\mathrm{I}^{-}$(at distances $\delta_{1}$ and $\delta_{2}$ ) in a bent structure. In the solid state, this symmetry is distorted, due to the cations and the interactions with other $\mathrm{I}_{3}{ }^{-}$and $\mathrm{I}_{2}$ moieties. We explored the potential energy surface of the system (see Fig. 3) and analysed electron density indicators along the transformation path leading to $\mathrm{I}_{5}^{-}$. Fig. 3 shows that there is a similarity between the in vacuo potential energy surface and the one retrieved from the experimental crystal structures within the framework of structure correlation, ${ }^{[36]}$ although, as expected, the distances in the solid are typically shorter than in vacuo. In fact, one can easily observe an ideal translation of the crystal potential energy surface along the diagonal of the diagram in Fig. 3.
The effect of the mechanical pressure is completely different: the compressed structures of TEAI significantly deviate from the transformation path of the in vacuo or crystal potential surface at ambient pressure. It seems that the HP structures would not converge towards a symmetric $I_{5}^{-}$. This reflects well the multiple $\mathrm{I}_{3}-\mathrm{I}_{2}$ interactions in the crystals that significantly perturb the picture of a localized $\mathrm{I}_{5}^{-}$moiety (when compressed) and anticipates the catenation.

If analyzing only atomic positions, the formation of a genuine covalent bond may not be identified. Even the electron density itself would provide only partial indication of the nature of the chemical bonding, especially under compression. Therefore, we used a decomposition of both the electron density (through Quantum Theory of Atoms in Molecules ${ }^{[37]}$ ) and the energy (through EDA and IQA ${ }^{[38]}$ ) to analyze the nature of this interaction as a function of $\delta_{1}$. EDA and IQA are different schemes to partition the bond energy into classic, exchange-correlation and kinetic terms. For $\mathrm{I}_{5}^{-}$the two methods provide the same picture (cf. Fig. 4): the classic (electrostatic) contribution always dominates and increases with $\delta_{1}$, whereas the non-classic (exchange) never exceeds $25 \%$ even at short $\delta_{1}$ distances. The exchange is larger than in $\mathrm{KI}$, but substantially smaller than in $\mathrm{I}_{2}$. Thus, the I-I bond in CT complexes is simultaneously of ionic and covalent nature, ${ }^{[39]}$ although the ionic one always dominates.

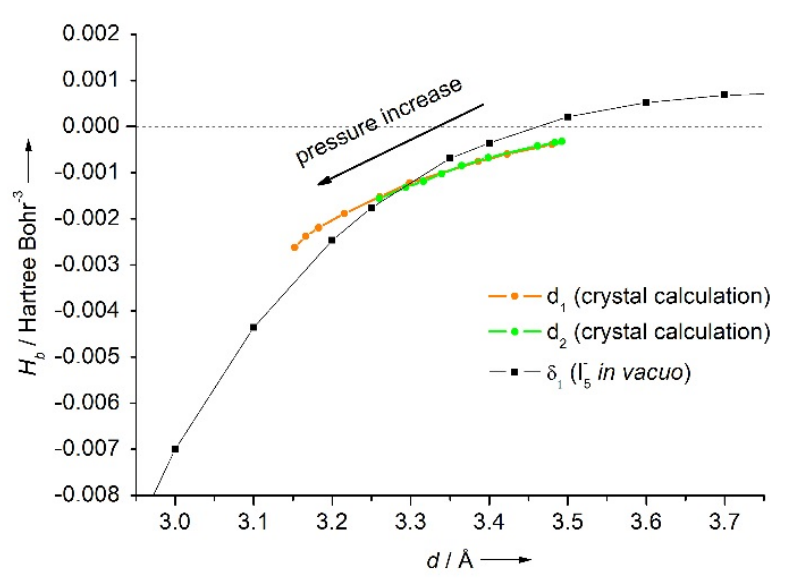

Figure 5: Green and orange: Energy density at the bond critical point (BCP) of the intermolecular contacts $d_{1}$ and $d_{2}$ calculated using the experimental structures. Black: Energy density at the BCP for the contact between $\mathrm{I}_{3}{ }^{-}$and $\mathrm{I}_{2}$ of $\mathrm{I}_{5}{ }^{-}$in vacuo calculated at different distances $\delta_{1}$.

Fig. 5 shows the behaviour of the total energy density at the $\mathrm{I}_{3}-\mathrm{I}_{2}$ BCP, proposed as criterion to identify a covalent bond:[40] a negative value implies that the potential energy locally dominates over the kinetic energy and thus that the electron density contributes to stabilization. Because of the homeomorphism between electron density and potential energy density[i], a negative energy density at $\mathrm{BCP}$ implies that the energy density is negative along the whole path interconnecting the valence shells of the interacting atoms. As shown in Fig. 5, the energy density at the BCP turns positive for $\delta_{1}>3.4 \AA$. For the experimental structures at different pressures, the trend mimics $I_{5}{ }^{-}$in vacuo both for $d_{1}$ (forming $I_{7}^{-}$) and $d_{2}$ (forming the extended chain). Thus, as 
a weak $|\cdots|$ contact shrinks, the shared electron density increases, as also shown by the delocalization index in $\mathrm{I}_{5}^{-}$(Fig. 4), and becomes more stabilizing. Noteworthy, while the delocalization approaches one electron pair for $\mathrm{I}-\mathrm{I}$ distances close to that in $\mathrm{I}_{2}$, the energy decomposition assigns anyway the dominant role to the electrostatic term. Even in $I_{2}$ it exceeds $60 \%$ of the total bond energy, in keeping with calculations for other homopolar diatomic molecules ${ }^{[41]}$.

In TEAl, the pressure induced addition reactions occur apparently in a continuous way, without changing the electronic states of the constituting moieties. However, the formation of an extended polymer raises the question whether this may affect the transport properties of the compound. For this reason, we carried out measurements of the electric resistance in TEAl and observed a dramatic drop between measurements at 9.4 and $11.1 \mathrm{GPa}$. This semi-conductive state is established for $d_{1} \sim 3.18 \AA$ and $d_{2} \sim$ $3.32 \AA$. In this range of distances, the percentage of covalency contribution to the bond has reached ca. $20 \%$ and upon further shortening the increase is small (Fig. 4).

At variance from $\mathrm{X}$-ray diffraction experiments, the resistivity was measured in nano-alumina powder, a non-hydrostatic PTM. Nevertheless, powder diffraction measurements performed in the same conditions, show that TEAI behaves in nano-alumina as in a hydrostatic medium and therefore the structure-property correlation (although not in situ) is reliable. The van der Pauw method $^{[42]}$ enables to estimate the sheet resistance of TEAI, which is $1.36 \Omega \cdot \mathrm{cm}$ at the highest pressure. Temperature scans in the range $25-55^{\circ} \mathrm{C}$ (see Figure S7) showed that the variation of the electrical conductivity (inversely proportional to the resistivity) in TEAl under high-pressure obeys the usual semiconductor equation: $\sigma=A e^{-E_{g} / 2 k T}$, where $\mathrm{E}_{\mathrm{g}}$ is the band gap and $A$ is a constant. Calculations prove a continuous band gap closure upon compression, in accordance with the measured decrease of resistivity.

As reported above, the semiconducting behavior is triggered at I-I distances for which the calculated covalent contribution to the bond is $20 \%$ (Fig. 4), which does not grow significantly upon further compression. On the other hand, the electron sharing continuously grows as the I-I distance decreases, which causes the valence band broadening and consequent band gap reduction, which seems continuous along the compression path. Noteworthy, only the gradual changes induced by pressure enable us to appreciate the structure-property correlation in such detail.

Recently, it was shown that PI also exhibit photoconductive properties, characteristic for CT complexes. ${ }^{[17]}$ This phenomenon, however, was not detected in TEAl under high-pressure, neither upon irradiation with a $1 \mathrm{~mW} 532 \mathrm{~nm}$ laser, nor with a $250 \mathrm{~W}$ tungsten lamp.

In this paper, we studied the effects of applying pressure on a solid state polyiodide. The compression stimulates the approach between complementary $\mathrm{I}_{3}^{-}$and $\mathrm{I}_{2}$ units, which are clearly separated and interacting mainly electrostatically at ambient pressure, whereas the covalent contribution increases upon compression, as revealed by theoretical calculations. Ultimately, this leads to the formation of CT chains, which explains the drastically increased conductivity.

These features make TEAl a tunable pressure-sensitive electric switch. Structural studies at high pressure can rationalize the synthesis and search for future organic and hybrid semiconductors based on PI. This includes structures with soft $|\cdots|$ contacts, which are the most prone for charge transport across the crystal under increased pressure. The results of this study indicate that solid PI may be used as solid electrolytes in dye-sensitized solar cells, which eliminates the need for organicbased gelators ${ }^{[43]}$ and ionic liquids in general.

\section{Experimental Section}

TEAI was synthesized according to the literature procedures ${ }^{[26]}$

Single-crystal diffraction measurements. A prismatic crystal of TEAI with approximately $200 \times 100 \times 30 \mu \mathrm{m}$ dimension was loaded in a MerrillBassett DAC, equipped with $0.5 \mathrm{~mm}$ diamonds and steel gaskets. Nitrogen was used as pressure-transmitting medium, and the pressure was calibrated with ruby fluorescence ${ }^{[44]}$. X-ray diffraction was measured the Material Science Beamline at Swiss Light Source $(\lambda=0.49471 \AA)^{[45]}$. Structures were refined with Shelx ${ }^{[46]}$ incorporated in OLEX2 ${ }^{[47]}$. All noniodine atoms were refined isotropically. Hydrogen atoms attached to carbons were geometrically placed and refined using a riding model. CCDC 1890310-1890317 (HP structures) and CCDC 1890330 (lowtemperature structure) contain the supplementary crystallographic data for this paper. These data are provided free of charge by The Cambridge Crystallographic Data Centre.

Electrical conductivity measurements. Two crystals of TEAI with $60 \times 60 \times$ $80 \mu \mathrm{m}$ size were loaded subsequently in DAC. A mixture of $\alpha$-nanoalumina and epoxy glue 4:1 was used as pressure transmitting medium. Pressure was calibrated using ruby fluorescence method. Four leads of golden wire were pressed against the sample (Fig. $1 \mathrm{SI}$ ) and the electrical resistivity was measured using the four-probe method with a Keithley 2450 multimeter.

CRYSTAL 14 ${ }^{[48,49]}$ was used for geometry optimizations and single point calculations in the crystal, using the B3LYP functional and Grimme dispersion correction ${ }^{[50]}$ ( for all atoms except iodine). The basis sets used were of POB_TZVP quality ${ }^{[51],[52]}$. No imaginary frequency was calculated. Properties in the crystal were obtained with TOPOND. Gaussian16 ${ }^{[53]}$ was used for geometry optimizations and single point calculations in vacuo at B3LYP/ 6-311G* level of theory[54].

AIMAll 14.11.23 $3^{[55]}$ was used for analysis of the electron density descriptors and IQA.

$A F^{[56]}$ with $B 3 L Y P / T Z P$, without frozen core and including scalar relativity was used for EDA.

\section{Acknowledgements}

We thank Dr. Michał Andrzejewski for support during the electrical measurements, the Frankfurt project of the DFG research unit FOR 2125 CarboPaT (Project WI1232) and the Swiss National Science Foundation (Project Nr. 162861 and NCCR Marvel) for the financial support.

Keywords: bonding analysis $\cdot$ conducting materials $\cdot$ crystalline materials $\cdot$ high-pressure chemistry $\bullet$ polyiodide

[1] P. Pelletier, J. B. Caventou, Ann. Chim Phys. 1819, 10, 142.

[2] P. H. Svensson, L. Kloo, Chem. Rev. 2003, 103, 1649-1684.

[3] R. A. Hoyt, E. M. Remillard, E. D. Cubuk, C. D. Vecitis, E. Kaxiras, J. Phys. Chem. C 2017, 121, 609-615.

[4] H. Haller, S. Riedel, Zeitschrift fur Anorg. und Allg. Chemie 2014, 640, 1281-1291.

[5] L. Kloo, J. Rosdahl, P. H. Svensson, Eur. J. Inorg. Chem. 2002.

[6] G. A. Landrum, N. Goldberg, R. Hoffmann, J. Chem. Soc. - Dalt. 


\section{Trans. 1997, 3605-3613.}

[7] K. Lamberts, P. Handels, U. Englert, E. Aubert, E. Espinosa, CrystEngComm 2016, 18, 3832-3841.

[8] E. Bartashevich, I. Yushina, K. Kropotina, S. Muhitdinova, V. Tsirelson, Acta Crystallogr. Sect. B Struct. Sci. Cryst. Eng. Mater. 2017, 73, 217-226.

[9] D. Cremer, E. Kraka, Angew. Chemie Int. Ed. English 1984, 23, 627-628.

[10] O. A. Syzgantseva, V. Tognetti, L. Joubert, in J. Phys. Chem. A 2013, pp. 8969-8980.

[11] V. Oliveira, E. Kraka, D. Cremer, Phys. Chem. Chem. Phys. 2016 18, DOI 10.1039/C6CP06613E.

[12] B. Li, Z. Nie, M. Vijayakumar, G. Li, J. Liu, V. Sprenkle, W. Wang, Nat. Commun. 2015, 6, 6303.

[13] N. H. Hiroshi Abe, Hiroaki Kishimura, Mayumi Takaku, Mai Watanabe, Faraday Discuss. 2017, 0, 1-12.

[14] P. Coppens, Extended Linear Chain Compounds, Plenum Press, New York, 1982.

[15] A. Oza, Cryst. Res. Technol. 1982, 19, 697-707.

[16] Y. X. Ren, M. Liu, T. S. Zhao, L. Zeng, M. C. Wu, J. Power Sources 2017, 342, 9-16.

[17] H. Yu, L. Yan, Y. He, H. Meng, W. Huang, Chem. Commun. 2017, 53, 432-435.

[18] A. Varvoglis, in Hypervalent lodine Org. Synth. (Ed.: A. Varvoglis), Academic Press, London, 1997, pp. 1-7.

[19] A. Sengupta, E. L. Quitevis, M. W. Holtz, J. Phys. Chem. B 1997, 101.

[20] L. Alvarez, J. L. Bantignies, R. Le Parc, R. Aznar, J. L. Sauvajol, A. Merlen, D. MacHon, A. San Miguel, Phys. Rev. B - Condens. Matter Mater. Phys. 2010, 82.

[21] P. Vinet, J. Ferrantef, J. R. Smith, J. H. Rose, J. Phys. C Solid State Phys. 1986, 19, L467-L473.

[22] R. J. Angel, J. Gonzalez-Platas, M. Alvaro, Zeitschrift fur Krist. 2014, 229, 405-419.

[23] A. M. James, M. P. Lord, Macmillan's Chemical and Physical Data, Macmillan, London, 1992.

[24] C. R. Groom, I. J. Bruno, M. P. Lightfoot, S. C. Ward, Acta Crystallogr. Sect. B Struct. Sci. Cryst. Eng. Mater. 2016, 72, 171179.

[25] K.-F. Tebbe, T. Gilles, Acta Cryst. C 1993, 49, 2042.

[26] Y. Wang, Y. Xue, X. Wang, Z. Cui, L. Wang, J. Mol. Struct. 2014, 1074, 231-239.

[27] P. H. Svensson, M. Gorlov, L. Kloo, Inorg. Chem. 2008, 47, 1146411466.

[28] M. D. García, J. Martí-Rujas, P. Metrangolo, C. Peinador, T. Pilati, G. Resnati, G. Terraneo, M. Ursini, CrystEngComm 2011, 13, 4411.

[29] C. He, D. A. Parrish, J. M. Shreeve, Chem. - A Eur. J. 2014, 20, 6699-6706.

[30] A. Gräfe-Kavoosian, S. Nafepour, K. Nagel, K.-F. Tebbe, Z. Naturforsch 1998, 53b, 641-652.

[31] Mizuno et al., J. Phys. Chem. 1981, 85, 1789-1794.

[32] X. An, J. Sun, Z. Lu, F. Ma, G. Zhang, Ceram. Int. 2017, 43, 6626-
6630.

[33] N. García, P. Esquinazi, J. Barzola-Quiquia, S. Dusari, New J. Phys 2012, 14, 0-14.

[34] A. Möller, T. Taetz, N. Hollmann, J. A. Mydosh, V. Kataev, M. Yehia, E. Vavilova, B. Büchner, Phys. Rev. B - Condens. Matter Mater. Phys. 2007, 76, 1-9.

[35] S. Klotz, J. C. Chervin, P. Munsch, G. Le Marchand, J. Phys. D. Appl. Phys. 2009, 42.

[36] H. B. Bürgi, J. D. Dunitz, in Struct. Correl., 2008, pp. 163-204.

[37] R. F. W. Bader, Atoms in Molecules: A Quantum Theory, International Series of Monographs on Chemistry, 1994.

[38] S. Racioppi, R. Della Pergola, V. Colombo, A. Sironi, P. Macchi, J. Phys. Chem. A 2018, 122, 5004-5015.

[39] A. M. Pendás, E. Francisco, M. A. Blanco, in Phys. Chem. Chem. Phys., 2007, pp. 1087-1092.

[40] D. Cremer, E. Kraka, Croat. Chem. Acta 1985, 57, 1259-1281.

[41] A. Kovács, C. Esterhuysen, G. Frenking, Chem. - A Eur. J. 2005, 11, 1813-1825.

[42] L. J. van der Pauw, Philips Tech. Rev. 1958, 20, 220-224.

[43] W. Kubo, K. Murakoshi, T. Kitamura, S. Yoshida, M. Haruki, K. Hanabusa, H. Shirai, Y. Wada, S. Yanagida, J. Phys. Chem. B 2001, 105, 12809-12815.

[44] H. K. Mao, J. Xu, P. M. Bell, J. Geophys. Res. 1986, 91, 4673.

[45] P. R. Willmott, D. Meister, S. J. Leake, M. Lange, A. Bergamaschi, M. Böge, M. Calvi, C. Cancellieri, N. Casati, A. Cervellino, et al., J. Synchrotron Radiat. 2013, 20, 667-682.

[46] G. M. Sheldrick, Acta Crystallogr. Sect. C Struct. Chem. 2015, 71, 3-8.

[47] O. V. Dolomanov, L. J. Bourhis, R. J. Gildea, J. A. K. Howard, H. Puschmann, J. Appl. Crystallogr. 2009, 42, 339-341.

[48] R. Dovesi, A. Erba, R. Orlando, C. M. Zicovich-Wilson, B. Civalleri, L. Maschio, M. Rérat, S. Casassa, J. Baima, S. Salustro, et al., Wiley Interdiscip. Rev. Comput. Mol. Sci. 2018, 8, 1-36.

[49] R. Dovesi, R. Orlando, A. Erba, C. M. Zicovich-Wilson, B. Civalleri, S. Casassa, L. Maschio, M. Ferrabone, M. De La Pierre, P. D'Arco, et al., Int. J. Quantum Chem. 2014, 114, 1287-1317.

[50] S. Grimme, J. Comput. Chem. 2006, 27, 1787-1799.

[51] J. Laun, D. Vilela Oliveira, T. Bredow, J. Comput. Chem. 2018, 39, 1285-1290.

[52] M. F. Peintinger, D. V. Oliveira, T. Bredow, J. Comput. Chem. 2013, 34, 451-459.

[53] G. A. Frisch, M. J., Trucks, G. W., Schlegel, H. B., Scuseria, G. E., Robb, M. A., Cheeseman, J. R., Scalmani, G., Barone, V., Petersson, Gaussian, Inc., Wallingford, CT 2016.

[54] M. N. Glukhovtsev, A. Pross, M. P. McGrath, L. Radom, J. Chem. Phys. 1995, 103, 1878-1885.

[55] T. A. Keith, TK Gristmill Software, Overl. Park KS, USA 2017.

[56] E. J. Baerends, T. Ziegler, ADF2017, SCM, Theoretical Chemistry, Vrije Universiteit, Amsterdam, The Netherlands 


\section{WILEY-VCH}

\section{COMVINCATION}

Entry for the Table of Contents (Please choose one layout)

Layout 1:

\section{COMMUNICATION}

Polymerization of a polyiodide salt is observed upon compression. The formation of infinite chains trigger the electrical conductivity at 10 GPa.

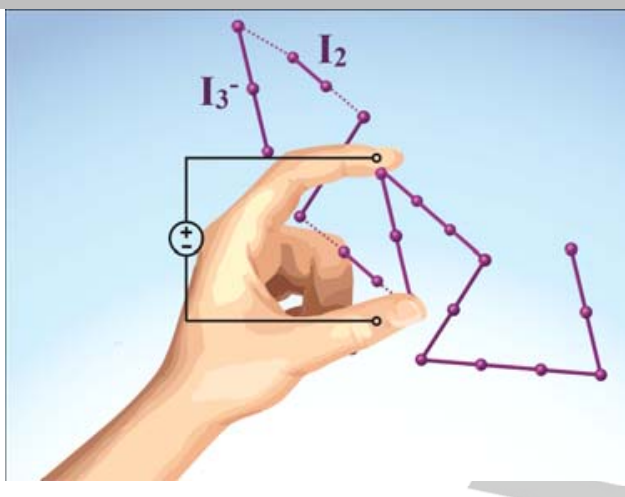

Tomasz Poręba,Michelle Ernst,Dominik Zimmer, Piero Macchi* and Nicola Casati* $^{*}$

Page No. - Page No.

Pressure-induced Polymerization and Electrical Conductivity of a Polyiodide

Layout 2:

\section{COMMUNICATION}

\section{((Insert TOC Graphic here))}

Author(s), Corresponding Author(s)*

Page No. - Page No.

Title

Text for Table of Contents 